\title{
Role of an ER stress response element in regulating the bidirectional promoter of the mouse CRELD2 - ALG12 gene pair
}

\author{
Kentaro Oh-hashi ${ }^{*}$, Hisashi Koga ${ }^{2}$, Shun Ikeda ${ }^{2}$, Kiyo Shimada ${ }^{2}$, Yoko Hirata ${ }^{1}$, Kazutoshi Kiuchi ${ }^{1}$
}

\begin{abstract}
Background: Recently, we identified cysteine-rich with EGF-like domains 2 (CRELD2) as a novel endoplasmic reticulum (ER) stress-inducible gene and characterized its transcriptional regulation by ATF6 under ER stress conditions. Interestingly, the CRELD2 and asparagine-linked glycosylation 12 homolog (ALG12) genes are arranged as a bidirectional (head-to-head) gene pair and are separated by less than $400 \mathrm{bp}$. In this study, we characterized the transcriptional regulation of the mouse CRELD2 and ALG12 genes that is mediated by a common bidirectional promoter.

Results: This short intergenic region contains an ER stress response element (ERSE) sequence and is well conserved among the human, rat and mouse genomes. Microarray analysis revealed that CRELD2 and ALG12 mRNAs were induced in Neuro2a cells by treatment with thapsigargin (Tg), an ER stress inducer, in a timedependent manner. Other ER stress inducers, tunicamycin and brefeldin A, also increased the expression of these two mRNAs in Neuro2a cells. We then tested for the possible involvement of the ERSE motif and other regulatory sites of the intergenic region in the transcriptional regulation of the mouse CRELD2 and ALG12 genes by using variants of the bidirectional reporter construct. With regards to the promoter activities of the CRELD2-ALG12 gene pair, the entire intergenic region hardly responded to Tg, whereas the CRELD2 promoter constructs of the proximal region containing the ERSE motif showed a marked responsiveness to Tg. The same ERSE motif of ALG12 gene in the opposite direction was less responsive to $\mathrm{Tg}$. The direction and the distance of this motif from each transcriptional start site, however, has no impact on the responsiveness of either gene to Tg treatment. Additionally, we found three putative sequences in the intergenic region that antagonize the ERSE-mediated transcriptional activation.

Conclusions: These results show that the mouse CRELD2 and ALG12 genes are arranged as a unique bidirectional gene pair and that they may be regulated by the combined interactions between ATF6 and multiple other transcriptional factors. Our studies provide new insights into the complex transcriptional regulation of bidirectional gene pairs under pathophysiological conditions.
\end{abstract}

\section{Background}

Among eukaryotes, analyses of the human and mouse genomes revealed that more than $10 \%$ of the genes are arranged as bidirectional gene pairs that are separated by less than only $1 \mathrm{~kb}$ of genomic DNA [1-3]. Some of these gene pairs could have evolved from a common ancestral gene during its duplication. Other gene pairs,

\footnotetext{
* Correspondence: oohashi@gifu-u.ac.jp

'Department of Biomolecular Science, Faculty of Engineering, Gifu University,

1-1 Yanagido, Gifu 501-1193, Japan

Full list of author information is available at the end of the article
}

however, do not have any genetic relationship between each other, and they are thought to play different biological functions within cells. It has been reported that the human PACPG-PARK2 gene pair [4], the human PREPL-C2ORF34 gene pair [5], the mouse surfeit Surf1Surf2 gene pair [6] and the mouse Sars2-Mrps12 gene pair [7] are co-regulated by distinctive transcriptional factors such as NRF-2, YY-1 or NF-Y. The transcriptional regulation of many other eukaryotic bidirectional gene pairs, however, remains to be determined.

\section{() Biomed Central}


Recently, we identified CRELD2 as a novel ER stressinducible gene by a microarray analysis of Tg-sensitive genes in Neuro2a cells and characterized the 5'upstream promoter region of the mouse CRELD2 gene [8]. Some pathophysiological conditions are reported to disrupt ER functions (e.g., the folding and modifying of newly synthesized transmembrane and secretory proteins) due to an accumulation of unfolded proteins $[9,10]$. The accumulation of unfolded proteins activates the expression of various genes through three resident ER stress sensors, PERK [11], IRE [12] and ATF6 [13]. The activation of these genes results in various outcomes (e.g., the refolding and/or degradation of accumulated proteins in the ER, and the activation of apoptotic signaling cascades). One of these ER stress sensors, ATF6, directly regulates the transcription of the CRELD2 gene via the ERSE motif, an ATF6 consensus sequence, located in its promoter [8]. The nucleotide sequence around the ERSE in the CRELD2 promoter is highly conserved within the mouse, rat and human genes. Interestingly, further genomic analyses reveal that the $A L G 12$ gene, one of the mannosyltransferase genes [14], is adjacent to the CRELD2 gene in a head-to-head configuration on the chromosome in some species. In this study, we first investigated the transcriptional regulation of the bidirectional CRELD2-ALG12 gene pair as $E R$ stress-inducible genes. We especially focused on evaluating the role of the ERSE motif, which is located within the 360-bp intergenic region, in regulating the expression of both genes under ER-stress conditions.

\section{Results}

ER stress induced the expression of both CRELD2 and ALG12 mRNAs in Neuro2a cells

Microarray analyses revealed that both CRELD2 and ALG12 mRNAs are induced in Tg-treated cells as well as GADD153 [15], Tib3 [16] and Herpud1 [17] mRNA, which are known ER stress-inducible genes (Table 1). To verify the Tg-induced expression of CRELD2 and ALG12 mRNAs in detail, Neuro2a cells were exposed to $0.1 \mu \mathrm{M}$ Tg for 4,8 , or $12 \mathrm{~h}$, and the expression of CRELD2, ALG12, GRP78 and GADD153 mRNAs were measured by RT-PCR. As shown in Figure 1A, CRELD2 and ALG12 mRNAs, as well as GRP78 [18] and GADD153 mRNAs, were up-regulated from 4 to $12 \mathrm{~h}$ after Tg-treatment. Next we examined the effects of other ER stress-inducing reagents (Tm and BFA), as well as serum withdrawal, on CRELD2 and ALG12 mRNA expression in Neuro2a cells. Like Tg treatment, those with Tm and BFA, but not serum withdrawal, induced CRELD2, ALG12, GRP78 and GADD153 mRNA expression similarly (Figure 1B).

\section{Comparison of the intergenic sequences of the CRELD2- $A L G 12$ gene pair within the mouse, rat and human genes} Next we analyzed the intergenic sequences of the CRELD2ALG12 gene pair within the mouse, rat and human genes. As shown in Figure 2, the nucleotide sequence of the mouse gene pair is highly homologous to that of the rat gene pair. The proximal promoter regions of the human and mouse CRELD2 genes, especially around the ERSE motif, are also well conserved. We then measured the basal promoter activities of the mouse CRELD2-ALG12 gene pair by using luciferase reporter constructs inserted with either the entire intergenic region $(-396 \sim+11)$ or the intergenic region containing various deletion mutations in either direction. As shown in Figure 3A, reporter constructs containing the entire intergenic region in either direction (+11/-396; CRELD2 promoter and $-396 /+11$; ALG12 promoter) showed the higher basal promoter activity. The activity of $A L G 12$ promoter $(-211 /+11)$ is still high in the absence of the ERSE motif, however a further deletion from position -211 to -108 in this promoter remarkably decreased its basal activity in Neuro2a cells. Furthermore, a deletion from position -136 to -228 in the CRELD2 promoter dramatically decreased CRELD2 promoter activity even though the ERSE motif is present. The deletion of a region around the ERSE motif $(-229 \sim-254)$ further decreased the promoter activity.

The role of the ERSE motif in CRELD2 and ALG12 promoter activities under ER stress condition

As shown in Figure 3B, the mouse CRELD2 promoter containing the proximal region $(-229 \sim-396)$, but no

Table 1 Tg-inducible genes in Neuro2a cells

\begin{tabular}{|c|c|c|c|c|}
\hline \multirow[t]{2}{*}{ Genes } & \multirow[t]{2}{*}{ Name } & \multirow[t]{2}{*}{ RefSeq No. } & \multicolumn{2}{|c|}{ Fold induction } \\
\hline & & & $4 \mathrm{~h}$ & $8 \mathrm{~h}$ \\
\hline ddit3 (GADD153) & DNA-damage inducible transcript 3 & NM_007837 & 6.1 & 9.8 \\
\hline trib3 & tribbles homolog 3 & NM_175093 & 3.9 & 7.2 \\
\hline herpud1 & homocysteine-inducible, endoplasmic reticulum stress-inducible, ubiquitin-like domain member 1 & NM_022331 & 4.4 & 6.0 \\
\hline creld2 & cysteine-rich with EGF-like domains 2 & NM_029720 & 2.9 & 4.3 \\
\hline $\operatorname{alg} 12$ & asparagine-linked glycosylation 12 homolog & NM_145477 & 3.6 & 3.7 \\
\hline
\end{tabular}

Neuro2a cells were treated with or without $0.1 \mu \mathrm{M}$ Tg for 4 or $8 \mathrm{~h}$. Total RNA was isolated from each cells and subjected to identify Tg-inducible genes using microarray analysis as described in methods. Representative genes are shown as ratio (Tg-treated cells/control cells). 


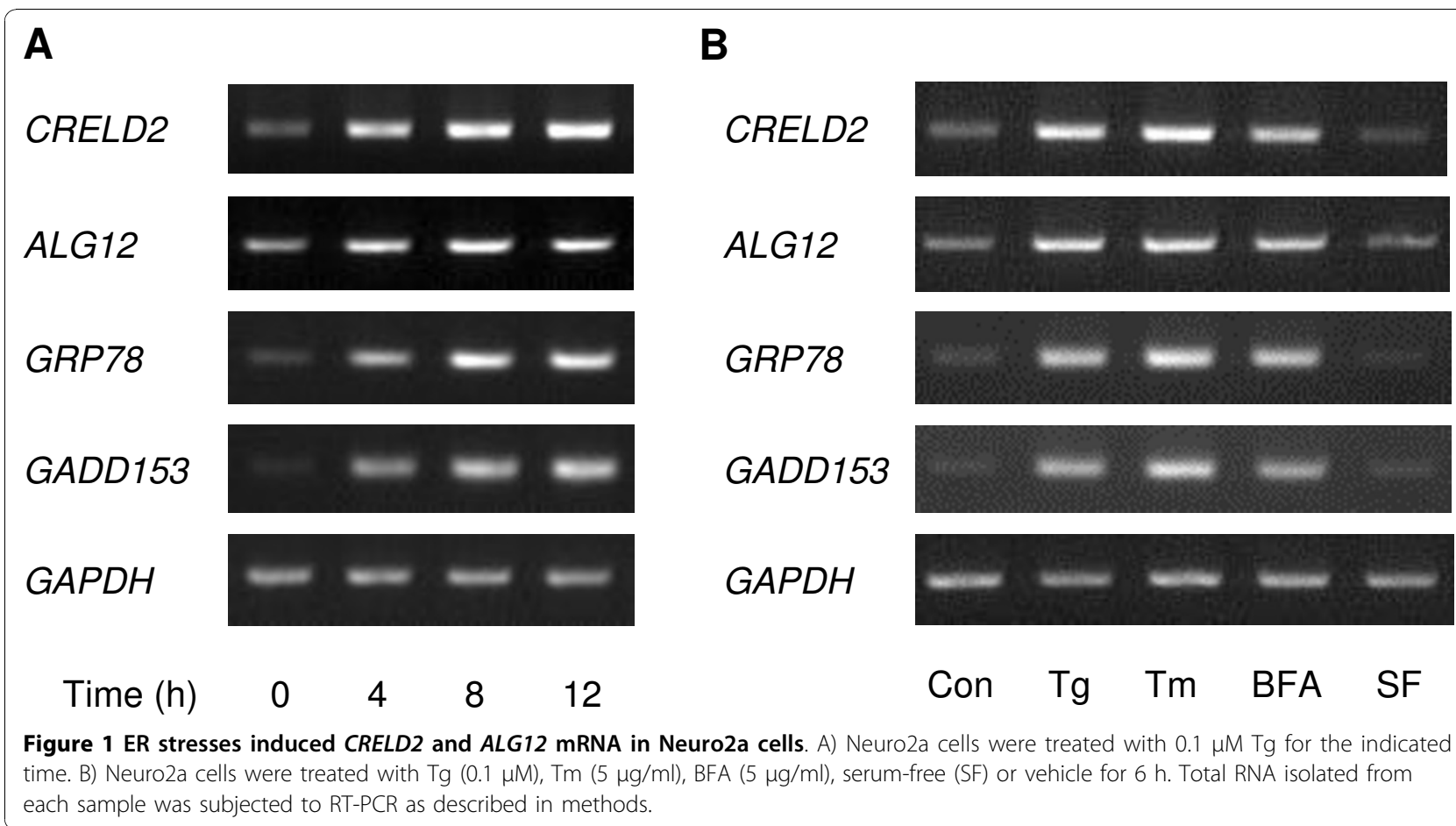

deletion mutation construct of mouse $A L G 12$ promoter, was significantly activated by Tg-treatment. Consistent with our previous report, the CRELD2 promoter construct containing the longer intergenic region $(-136 \sim$ -396) showed higher basal promoter activity but a lower responsiveness to $\mathrm{Tg}$ compared to the above-mentioned construct (-229/-396) (Figure 3B). The CRELD2 promoter without the ERSE motif $(-255 /-396)$ had an even further diminished basal promoter activity and Tgresponsiveness. Next, we determined the effect of various mutations within the ERSE motif on the activity of the mouse $A L G 12$ promoter. Unlike the CRELD2 promoter $(-229 /-396)$ and its point mutation constructs $(-229 /-396 \mathrm{~m} 1$ or $\mathrm{m} 2$; a mutation at the ATF6 or NF-Y binding site $[8,13,18-20]$, respectively), the mutations in the ALG12 promoter $(-361 /+11 \mathrm{~m} 1$ and $\mathrm{m} 2)$ did not affect the basal promoter activity and the responsiveness to Tg (Figure 4). Then, we evaluated the effect of the ERSE motif's direction on the responsiveness of the mouse CRELD2-ALG12 gene pair to Tg by using a pGL3 vector containing the SV40-promoter. The reporter constructs containing a partial intergenic region of the gene pair $(-343 \sim-212)$ in either direction responded to $\mathrm{Tg}$ and ATF6-overexpression similarly (Figure 5A and 5B). Interestingly, Tg-treatment and ATF6-overexpression stimulated the luciferase activity of the CRELD2 promoter (-229/-396) construct more effectively than the $A L G 12$ promoter $(-361 /+11)$ construct (Figure 5C and 5D).
To study the unresponsiveness of the $A L G 12$ promoter $(-361 /+11)$ to $\mathrm{Tg}$, we prepared another reporter construct in which the middle intergenic region of the ALG12 promoter $(-211 \sim-108)$ that contributes to the basal promoter activity is deleted (Figure 3A). This construct $(-343 /-212 \&-107 /+11)$, however, did not respond to Tg (Figure 6A). Serial deletions of the 3'-end of the ALG12 promoter lacking the middle intergenic region revealed that there is a suppressive site from position -75 to -16 in the ALG12 promoter (Figure 6B). Deletion around three putative Ets family binding sites (TTCC) $[21,22]$ from position -52 to -20 (Figure 2, site III) in the ALG12 promoter $(-343 /-212 \&-107 /+11 \mathrm{~m} 1)$ also restored responsiveness to $\mathrm{Tg}$. Yet, this same site III deletion in the ALG12 promoter containing the middle intergenic region $(-361 /+11 \mathrm{~m} 3)$ showed unresponsiveness to $\mathrm{Tg}$ (Figure 6C). To determine if there are other suppressive sites in this intergenic region, we prepared various deletion mutation constructs of the ALG12 promoter and evaluated their responsiveness to Tg. As shown in Figure 7A, we identified two additional suppressive sites (I and II in Figure 2). We also found that the deletion of all three sites $(-361 /+11 \mathrm{~m} 7$; ALG12 promoter) was required in order to restore the responsiveness to Tg. A mutation in either the NF-Y binding site (CCAAT) of the ERSE motif $(-361 /+11 \mathrm{~m} 8)$ or a site 8-bp downstream of the ERSE motif $(-361 /+11 \mathrm{~m} 9)$ in the $A L G 12$ promoter showed that each NF-Y binding site partially participated in its basal promoter activity. 


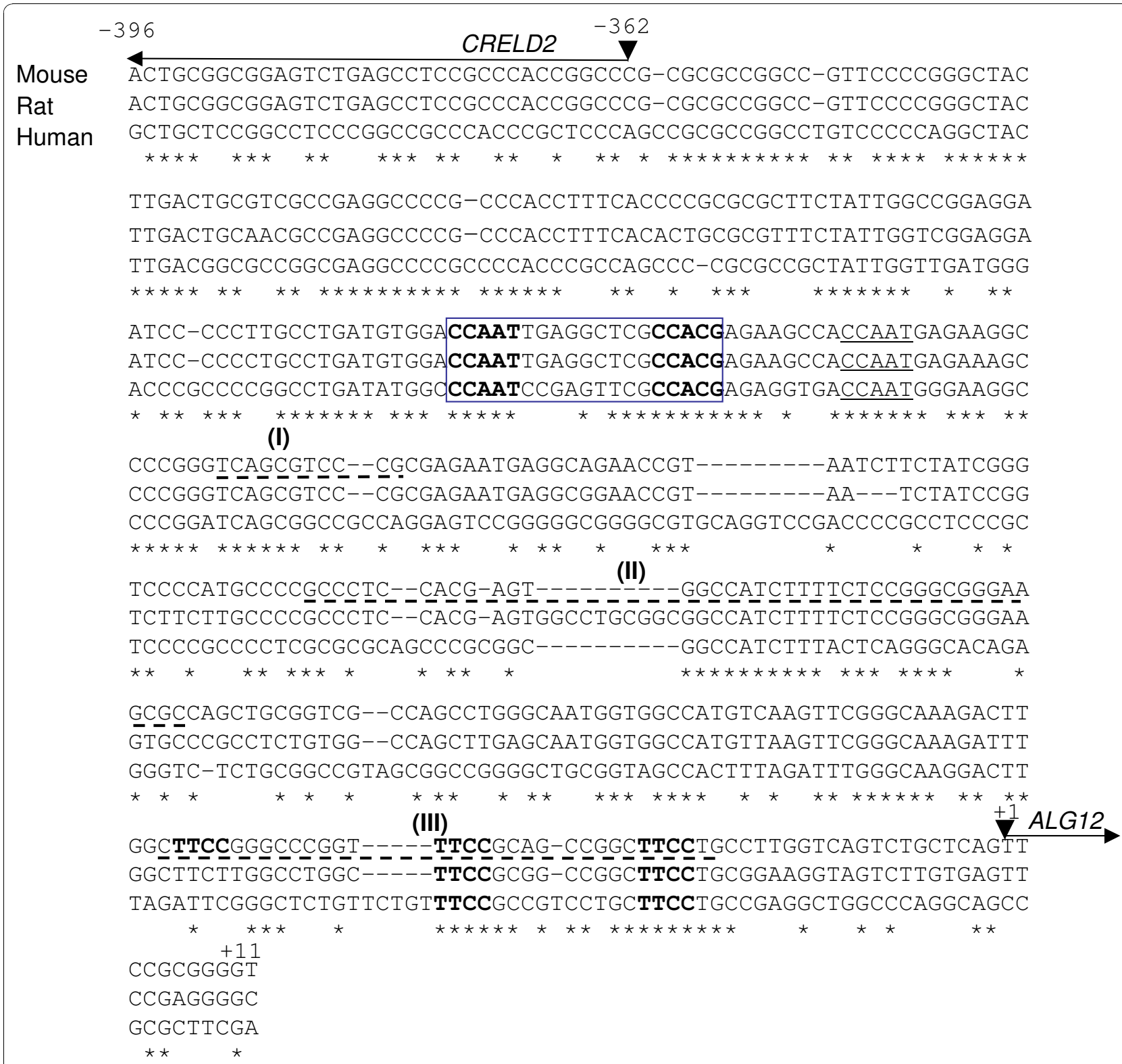

Figure 2 Nucleotide sequences of the mouse, rat and human CRELD2-ALG12 gene pairs. Nucleotide sequences conserved among the mouse, rat and human CRELD2-ALG12 gene pairs are shown with asterisks. A conserved ERSE motif and an adjacent NF-Y binding site are shown in a box and underline, respectively. The transcriptional direction and putative transcriptional start sites of the mouse CRELD2 and ALG12 genes are shown with arrows and arrowheads, respectively. Three suppressive regions identified in this study are shown with broken lines (sites I, II and III). Putative binding sequences of the Ets family (TTCC) in site III are shown with bold types.

Only the site in the ERSE motif in the $A L G 12$ promoter $(-361 /+11 \mathrm{~m} 8)$, however, are crucial to the responsiveness to $\mathrm{Tg}$ as well as the CRELD2 promoter $(-209 /+396$ $\mathrm{m} 1$ ) (Figure 7B and 7C). Finally, we measured the promoter activity of the entire intergenic region of the CRELD2-ALG12 gene pair in the both direction (-396/ +11; CRELD2 and ALG12 promoters) after Tg treatment or ATF6 cotransfection. Both promoter constructs only slightly responded to $\mathrm{Tg}$, but the deletion of the three suppressive regions $(+11 /-396 \mathrm{~m} 1$; CRELD2 promoter and -396/+11 m1; ALG12 promoter) restored responsiveness to Tg. Furthermore, the basal promoter activities of these mutant constructs markedly decreased (Figure 8A). ATF6-overexpression enhanced the promoter activity of all of the above-mentioned constructs. The Tg-responsive reporter constructs $(+11 /-396 \mathrm{~m} 1$ and -209/-396; CRELD2 promoters and $-396 /+11 \mathrm{~m} 1$; $A L G 12$ promoter) also showed a further increase in their promoter activities by ATF6-overexpression (Figure 8B). 


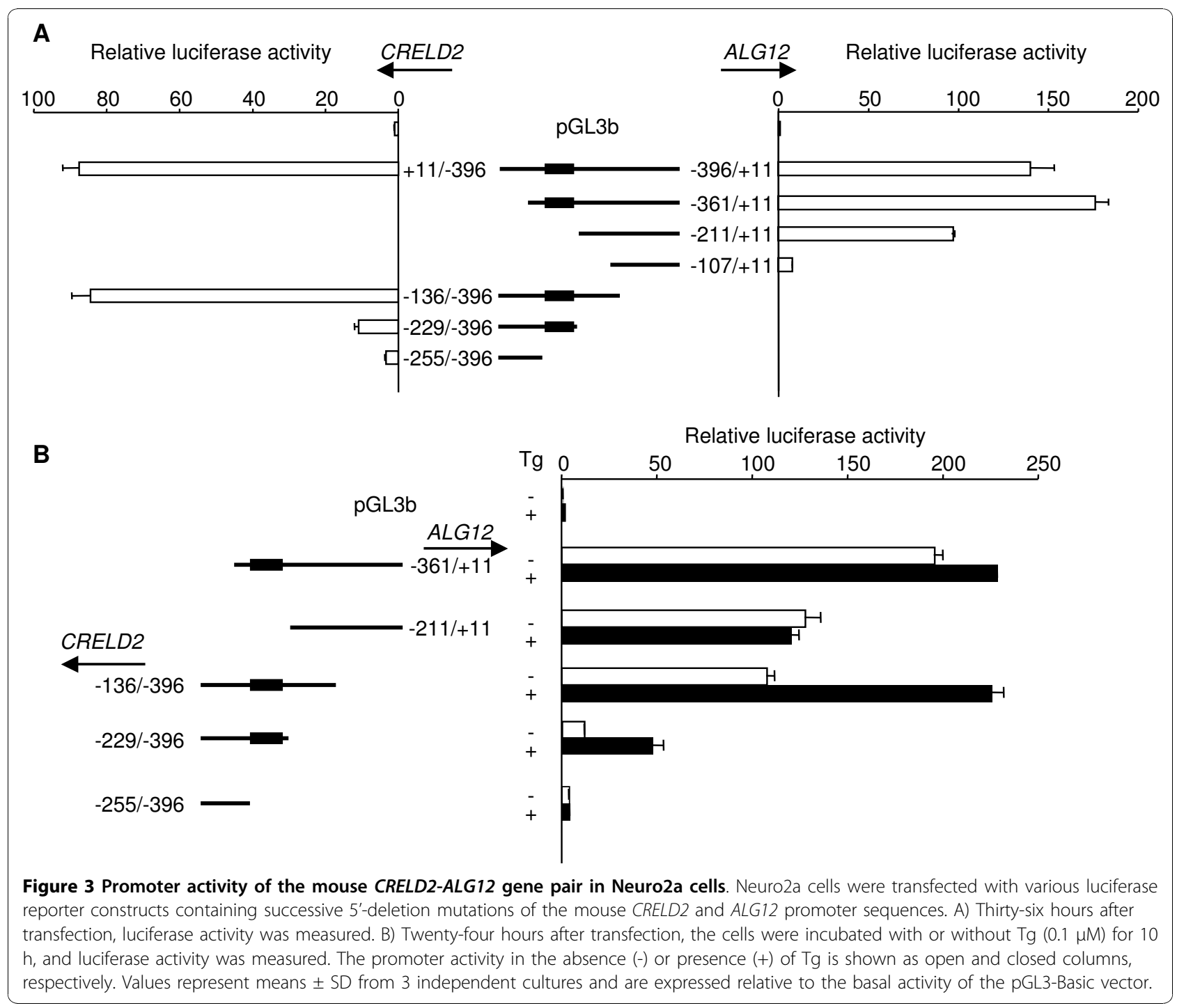

\section{Discussion}

Recently, we identified CRELD2 as a novel ER-stress inducible gene and characterized its ATF6-dependent transcriptional regulation using constructs containing the proximal region of the mouse CRELD2 promoter [8]. Genomic analyses reveal that the $A L G 12$ gene [14] is adjacent to the CRELD2 gene in a head-to-head configuration on the chromosome in some species. CRELD2 and $A L G 12$ genes are a bidirectional gene pair arranged less than $400 \mathrm{bp}$ apart. The nucleotide sequences of this intergenic region are moderately conserved among the mouse, rat and human genes. Furthermore, those regions around an ERSE motif in the CRELD2-ALG12 gene pair are highly conserved (Figure 2). In this study, we demonstrate that the expression of CRELD2 and $A L G 12$ mRNAs, and GRP78 and GADD153 mRNAs, which are well known ER stress-inducible genes, was induced by three distinct ER stress inducers (Figure 1). In regards to the promoter activity of the mouse CRELD2-ALG12 gene pair, only the CRELD2 promoter containing just the proximal region (-229/-396 and -209/-396; CRELD2 promoter) significantly responded to Tg. Additionally, the CRELD2 promoter containing the full intergenic region $(+11 /-396$; CRELD2 promoter) decreased in responsiveness to $\mathrm{Tg}$, whereas its basal promoter activity markedly increased. In contrast, the ALG12 promoters only slightly responded to $\mathrm{Tg}$ even though some of the reporters contained the ERSE motif, which is 300-bp apart from the transcription start site of the mouse ALG12 gene. The direction of the ERSE motif and its distance from each of the transcription start sites for the mouse CRELD2 or $A L G 12$ genes, however, appear to have no influence in these findings. Therefore, it seems that the full intergenic region contains one or more unknown suppressive sites that interfere with the ERSE-mediating enhancement of the ALG12 and CRELD2 promoter activities. 


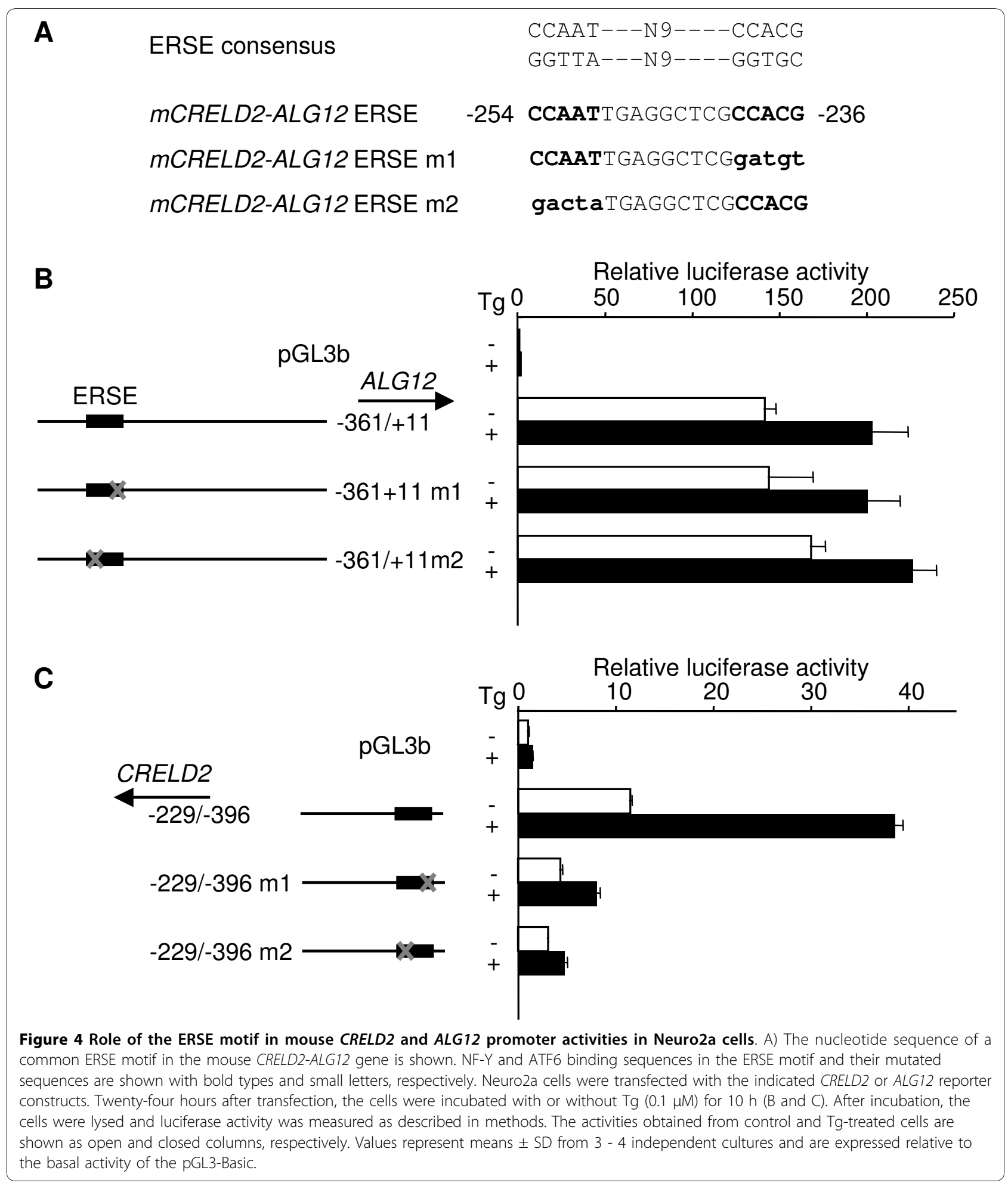

Reporter constructs used in this study contain 5'untranslated regions (5'-UTR) of CRELD2 and/or $A L G 12$ gene. Especially, reporter constructs containing the entire intergenic region of CRELD2 - ALG12 gene pair contain the UTR regions at both ends. However, the deletion of three suppressive sites (sites I, II and III) in each construct recovered the responsiveness to $\mathrm{Tg}$ $(-343 /-212--107 /+11 \mathrm{~m} 1$ in Figure $6 \mathrm{C}$ and $-361 /+11$ $\mathrm{m} 7,+11 /-396 \mathrm{~m} 1$ and $-396 /+11 \mathrm{~m} 1$ in Figure $7 \mathrm{~A}$ and $8 \mathrm{~A})$. Therefore, it seems likely that each 5'-UTR hardly 


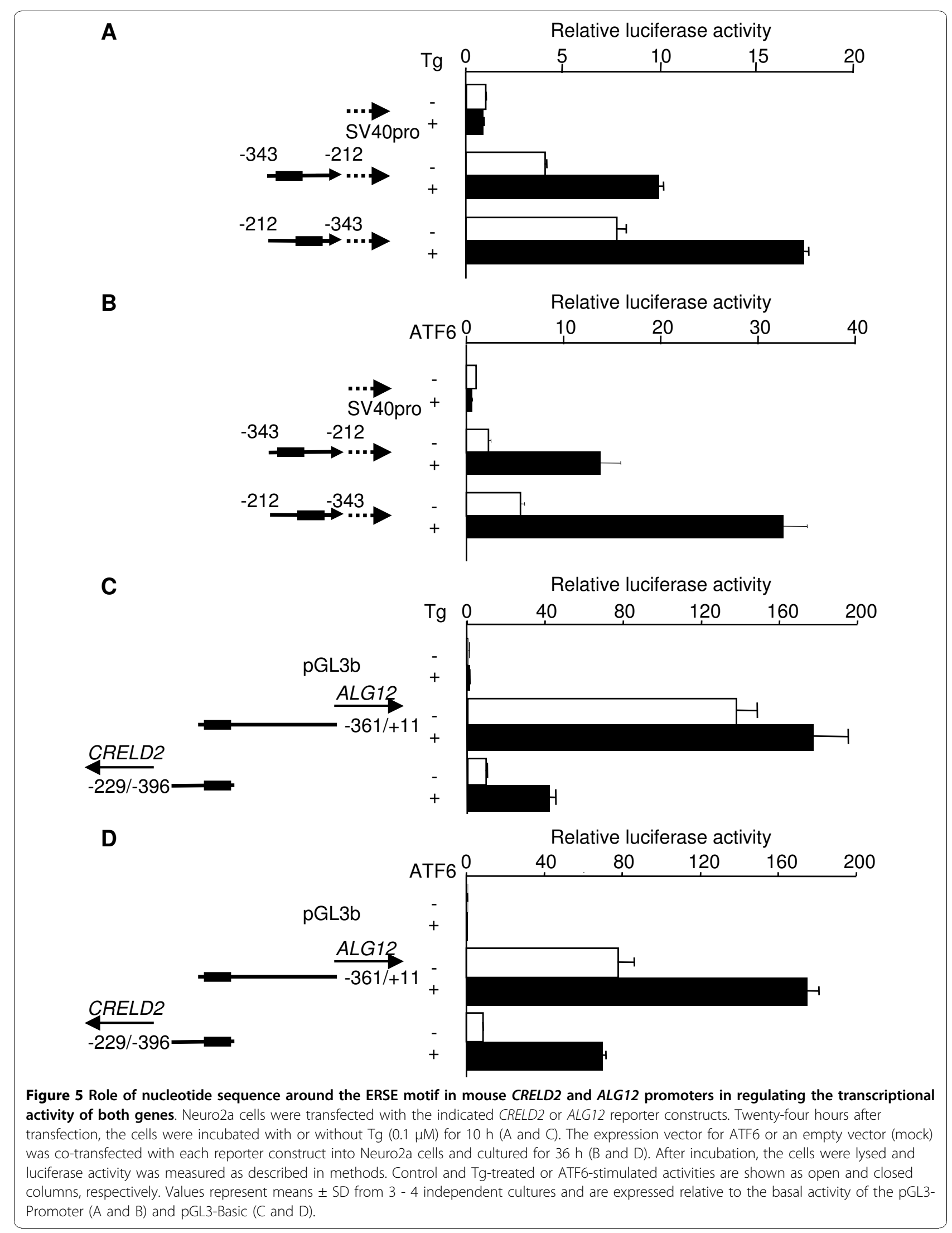




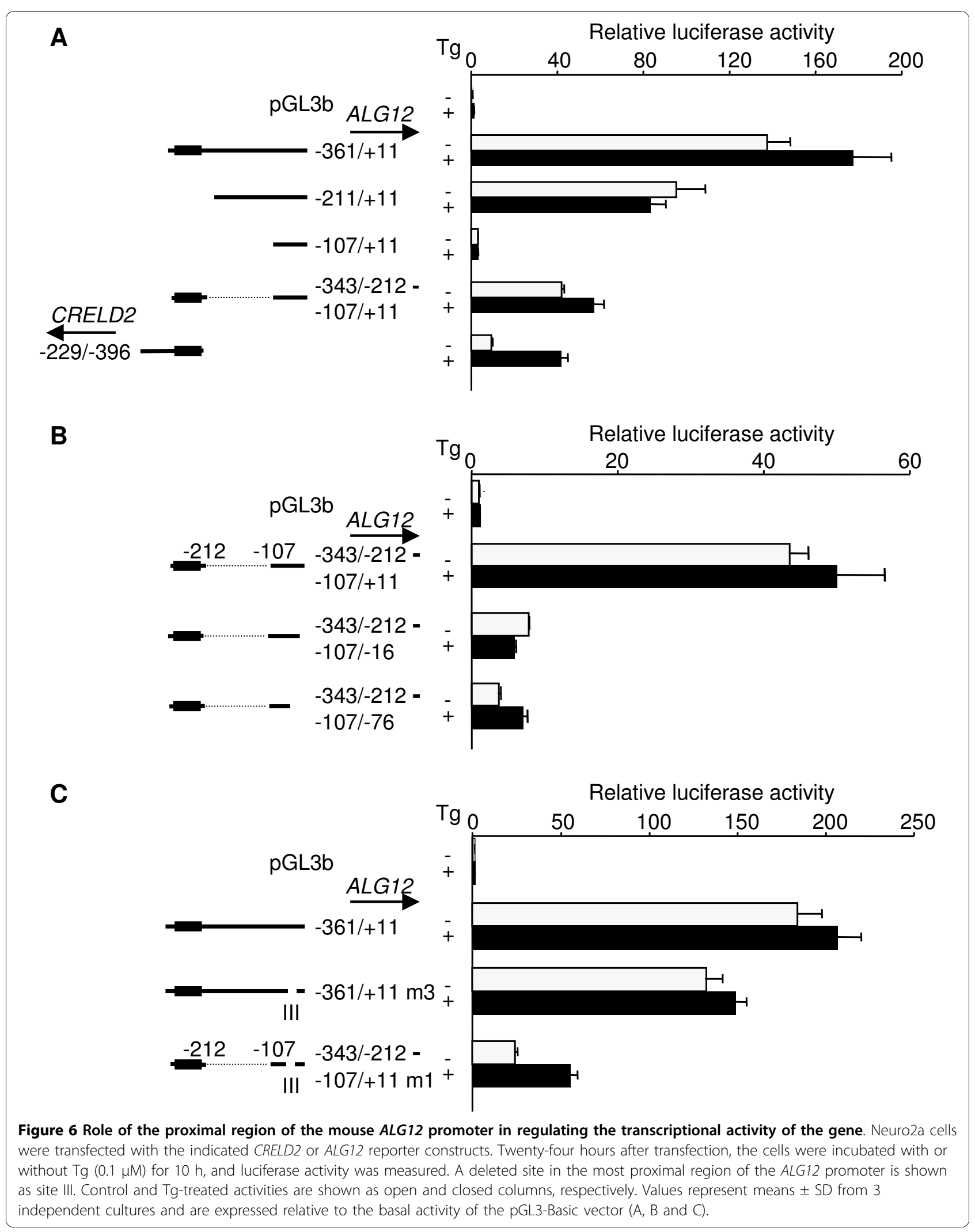




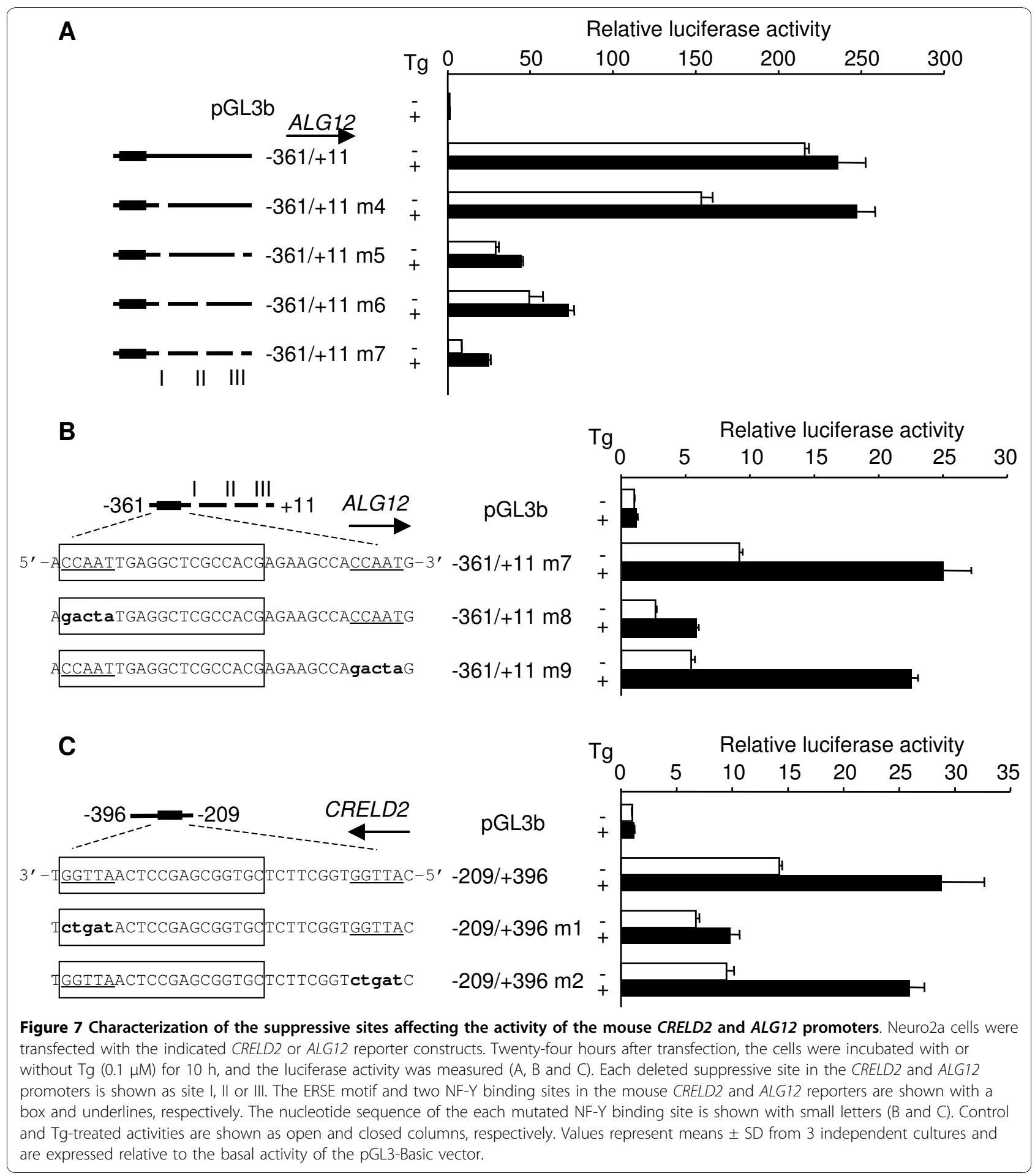

influenced the corresponding promoter activity of the CRELD2 and ALG12 promoter constructs in our assay system. CRELD2 (NM_029720.2) and ALG12 (NM_145477.1) genes possess 5'-UTR (66 and 55 bp) and 3'-UTR (245 and $584 \mathrm{bp}$ ) respectively though their effects on transcription are not elucidated yet. Further characterization of these regions would reveal regulations of CRELD2 and ALG12 mRNA expression.

Using various deletion mutation constructs, we showed that three suppressive sites in the CRELD2$A L G 12$ gene pair play a crucial role in interfering with $\mathrm{Tg}$ responsiveness. Interestingly, the deletion of all three 


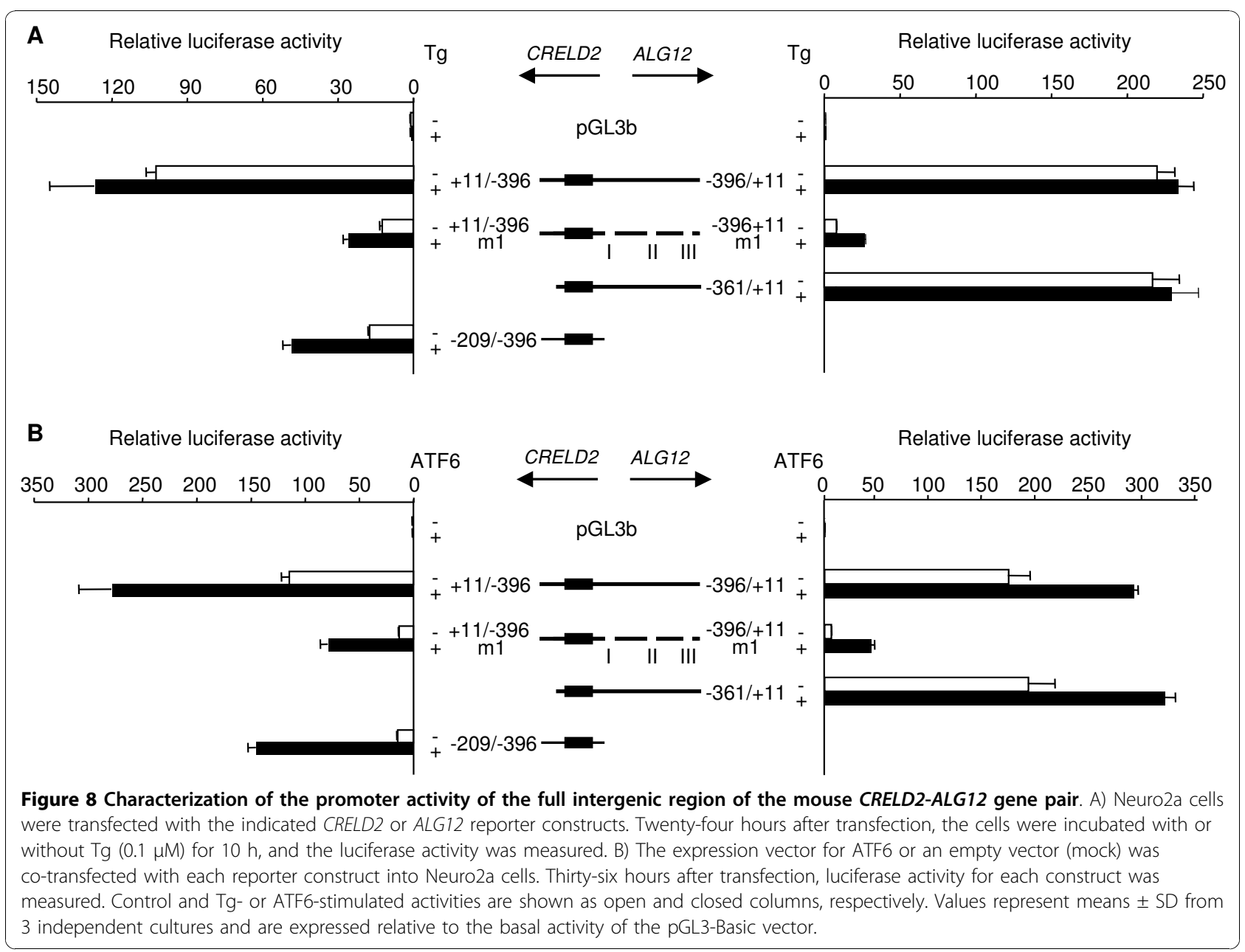

of these suppressive sites was required in order to restore the responsiveness to Tg (Figure 2, 7 and 8). These results imply that these suppressive sites are not only important in maintaining basal promoter activity, but that they synergistically counteract the ERSEmediated transcriptional activity (Figure 9). Among these sites, the most proximal to the ALG12 promoter contains a conserved response element that Ets-family transcriptional factors recognize [21]. Ets transcription factors consist of approximately 30 family members and share a highly conserved DNA-binding domain. It has been reported that these factors are involved in regulating a variety of biological processes including development, differentiation and inflammation $[21,22]$. In the site II, there are putative YY1- (GCCATC) [23] and MAZ- (CCCCGCCCT) [24] binding sites judged from some databases such as SwissRegulon (a database of genome-wide annotations of regulatory sites), but the precise roles remain to be determined. On the contrary,

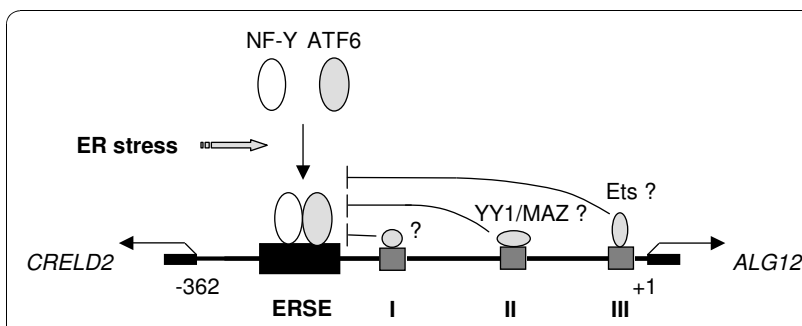

Figure 9 Proposed mechanisms regulating promoter activities in the intergenic region of CRELD2 and ALG12 gene pair. Under ER stress conditions, ATF6 and NF-Y translocate into nucleus, form complex and specifically bind the ERSE motif to induce CRELD2 and ALG12 promoter activities. Three sites (I, II and III) in the intergenic region identified in this study are important for their basal promoter activities. However, factors binding to the site I, II and III could antagonize the ERSE-mediated up-regulation of both promoter activities cooperatively. According to the database of transcription regulatory motifs, the site II contains putative YY1- and MAZbinding motifs and the site III contains an Ets family-binding motif, respectively. 
we are unable to find any unique sequences in the sites I. Further studies characterizing each of these suppressive sites (site I, II, and III) are required in order to understand the complex transcriptional regulation of the CRELD2-ALG12 gene pair. Jones PL et al. reported that murine manganese superoxide dismutase gene is regulated through a complex intronic enhancer involving $\mathrm{C} /$ EBP $-\beta$ and NF- $\kappa$ B [25]. Donati G et al. demonstrated that ER stress triggers dynamic modification of chromatin components and transcriptional factors under ER stress [20]. Therefore, we should focus on other aspects such as local chromatin remodeling and histone modifications (e.g., phosphorylation, acetylation and methylation of histones) within the CRELD2 and ALG12 genes in addition to the 5 '-flanking sequences in this intergenic region. Furthermore, other approaches should be employed to elucidate the discrepancy between the expression levels of both intrinsic mRNAs and the promoter activities of their full intergenic region under ER stress conditions.

Among the bidirectional gene pairs characterized in mammalian cells, Surf1-Surf2 [6], Reql4-Lrrc14 [26], PDCD10-SERPINI1 [27] and Thox-DUOXA [28] gene pairs seem to share their intergenic region equally because mutations in the transcription factor binding sites decline those promoter activities equally. In contrast, the transcriptional regulations of C2ORF34-PREPL [5], Sarsm-Mrps12 [7] and HAND2-DEIN [29] are asymmetric. According to the present study, the transcriptional regulatory pattern of the mouse CRELD2-ALG12 gene pair belongs to the latter group. Analyses of these bidirectional gene pair sharing a common intergenic region have mostly consisted of characterization without any stimuli. Recently, Zanotto E et al. reported that the Sarsm-Mrps12 promoter activity is modulated by mitochondrial stresses, especially mitochondrial reactive oxygen species, in a complex manner [30]. At this time, however, the significance and relevance of many bidirectional gene pairs under pathophysiological conditions are not well understood.

The mammalian ALG12 gene is the ortholog of the yeast gene that encodes the dolichyl-P-Man:Man GlcNAc $_{2}$-PP-dolichyl $\alpha 6$-mannosyltransferase, and its mutation causes a congenital disorder affecting glycosylation in the ER $[14,31,32]$. Clinically, a child suffering from a point mutation in the $A L G 12$ gene has been reported to show severe symptoms such as psychomotor retardation, hypotonia, growth retardation, dysmorphic features and anoxia [31]. Sequential protein glycosylation in the ER is important in maintaining the quality control of glycoproteins through folding and ER-associated protein degradation. Moreover, its defects could also interfere with the intracellular trafficking and secretion of glycoproteins. Therefore, suitable regulation of the ALG12 gene should be required in order to maintain ER homeostasis.

As the CRELD proteins have multiple EGF-like domains, they are considered to be cell adhesion molecules [33-35]. It has been reported that missense mutations in the CRELD1 gene increases an individual's susceptibility to atrioventricular septal defects [33], but the physiological roles of these family members remain poorly understood. In contrast to CRELD1, CRELD2 lacks a transmembrane domain in the C-terminal region. Ortiz et al. reported that the overexpression of CRELD2 impairs the membrane transport of acetylcholine receptor $\alpha 4 / \beta 2$ in Xenopus lae$v i s$ oocytes [36]. We recently demonstrated that the CRELD2 gene is one of the downstream targets of ATF6 and that its product is predominantly localized in the ER-Golgi apparatus [8]. Interestingly, the mouse model for multiple epiphyseal dysplasia, which specifically expresses a mutation in matrilin-3, was reported to induce CRELD2 mRNA expression and other ER-stress inducible genes as the symptoms progressed [37]. According to these reports, CRELD2 seems to be involved in the folding, processing and transport of some proteins under pathophysiological conditions, though the precise role of CRELD2 remains to be determined. Furthermore, we believe that the sharing of the ERSE motif in the CRELD2-ALG12 gene pair may be advantageous in regulating ER homeostasis under various ER-stress conditions, even though it is unlikely that the CRELD2 and ALG12 proteins function by directly interacting with each other.

\section{Conclusion}

In this study, we first demonstrate that both the CRELD2 and ALG12 genes, which form a bidirectional gene pair, are potent ER stress-inducible genes. Our present results indicate that the CRELD2-ALG12 gene pair could be asymmetrically regulated by multiple transcriptional factors in addition to ATF6. Because the CRELD2-ALG12 gene pair contains an evolutionally conserved ERSE motif, the cooperative induction of these genes may play important roles in confronting ER stresses and in appropriately regulating ER homeostasis and cell fates, together with other ER stress-inducible genes (e.g., GRP78 and GADD153). Therefore, further characterization of the CRELD2-ALG12 gene pair may provide new insights into the complex transcriptional regulation of ER stress-inducible genes as well as into the onset and progression of various ER stressassociated diseases.

\section{Methods}

\section{Cell culture and treatment}

Neuro2a cells were maintained in Dulbecco's Modified Eagles minimum essential Medium containing 8\% fetal bovine serum. Transfection of each construct used in 
this study was performed using Lipofectamine-Plus reagent (Invitrogen) according to the manufacturer's instructions. For stimulation, Neuro2a cells were treated with $\mathrm{Tg}(0.1 \mu \mathrm{M})$, Tm $(5 \mu \mathrm{g} / \mathrm{ml})$, BFA $(5 \mu \mathrm{g} / \mathrm{ml})$ or serum-free medium (SF) for the indicated time.

\section{Construction of plasmids}

For preparation of reporter constructs for the mouse CRELD2 and ALG12 promoters, genomic DNA from Neuro2a cells was extracted, and the mouse CRELD2 and $A L G 12$ promoters were amplified by polymerase chain reaction (PCR) and cloned into the pGL3-Basic vector (Promega). To evaluate the promoter activity of the intergenic region of the mouse CRELD2 and ALG12 genes, the position of the putative transcriptional start site of mouse CRELD2 or ALG12 is defined as -362 and +1 , respectively. The promoter region was defined using a database of the NIH full-length cDNA project and RIKEN functional annotation of a full-length mouse cDNA collection (FANTOM). To characterize the enhancer activity of the partial intergenic region containing ERSE (-343 -212), it was inserted into the pGL3-Promoter vector (Promega). We also constructed various other bidirectional reporter construct carrying point and deletion mutations. Mouse ATF6 was amplified by PCR using cDNA from Neuro2a cells and cloned into the pFlag-CMV vector.

Reverse transcription polymerase chain reaction (RT-PCR) To estimate the expression level of each gene by RT-PCR, total RNA was extracted from cells lysed with Trizol and converted to cDNA by reverse transcription using random ninemers to prime superscript III RNase ${ }^{-}$reverse transcriptase (RT) (Invitrogen) as previously described [8]. Specific cDNAs were mixed and amplified with a PCR reaction mixture (Taq PCR kit, Takara). The RT-PCR primers used in this study were as follows: CRELD2 sense primer 5'-ACTGAAGAAGGAGCACCCCAAC-3', CRELD2 antisense primer 5'-GCACACTCATCCACATCCACACA-3', ALG12 sense primer 5'-GTGATTTCTGGACTCTGGAC-3', ALG12 antisense primer 5'-GGGGT ATGAAGAGAAGGCTGCA-3', GADD153 sense primer 5'-GAATAACAGCCGGAACCTGA-3', GADD153 antisense primer 5'-GGACGCAGGGTCAAGAGTAG-3', GRP78 sense primer 5'-ACCAATGACCAAAACCGCCT3', GRP78 antisense primer 5'-GAGTTTGCTGATAAT TGGCTGAAC-3', GAPDH sense primer 5'-ACCACAG TCCATGCCATCAC-3', GAPDH antisense primer 5'-TC CACCACCCTGTTGCTGTA-3'. The typical reaction cycle conditions were $30 \mathrm{sec}$ at $96^{\circ} \mathrm{C}, 30 \mathrm{sec}$ at $60^{\circ} \mathrm{C}$ and $30 \mathrm{sec}$ at $72^{\circ} \mathrm{C}$. The results represent $18 \sim 33$ cycles of amplification, after which cDNAs were separated by electrophoresis on $2.0 \%$ agarose gels and visualized using ethidium bromide. Experiments were repeated to confirm reproducibility.

\section{GeneChip analysis}

After Neuro2a cells were incubated in the absence or presence of $\mathrm{Tg}$ for the indicated time, total RNA was extracted as described in the above methods. After measuring the quantity and quality of the RNA, biotinlabeled cRNAs were generated from $5 \mu$ g of each total RNA using a GeneChip ${ }^{\oplus}$ One-Cycle Target Labeling and Control Reagents package (Affymetrix) according to the manufacturer's protocol. Afterwards, $15 \mu \mathrm{g}$ of the purified cRNAs were mixed with $3 \mathrm{nM}$ Control Oligo B2, and the hybridization cocktail was denatured at $99^{\circ} \mathrm{C}$ for $5 \mathrm{~min}$ in a heat block, followed by incubation at $45^{\circ} \mathrm{C}$ for $5 \mathrm{~min}$, and centrifugation for $5 \mathrm{~min}$ in order to remove any insoluble material. Hybridization to a mouse DNA array (GeneChip Mouse Genome 4302.0 Array) was carried out at $45^{\circ} \mathrm{C}$ for $16 \mathrm{~h}$ using a hybridization oven 640 (Affymetrix). After hybridization, the arrays were washed and stained with the GeneChip ${ }^{\circ}$ Hybridization Wash and Stain Kit (Affymetrix) using the GeneChip ${ }^{\circ}$ Fluidics Station 450 (Affymetrix) according to the manufacturer's protocol. The signal intensities were quantified using a GeneArray Scanner 3000 (Affymetrix), and the raw data obtained were converted into MAS (Microarray Suite) files using the GeneChip Operating Software (GCOS). After normalization, the identification of the temporal expression patterns of genes was performed using the Spotfire ${ }^{\circ}$ DecisionSite. In this analysis, the mean signal intensity of gene expression in each group included in the study (three samples/group) was used. As a selection criteria to present only the most relevant genes, a cutoff of a 2.0-fold increased/ decreased expression and a $\mathrm{p}<0.01$ were arbitrarily chosen.

\section{Reporter gene assay}

Reporter constructs and the pRL-TK vector, an internal control, were transfected into Neuro2a cells in a 48-well plate. Twenty-four hours after transfection, the cells were treated with $\operatorname{Tg}(0.1 \mu \mathrm{M})$ or vehicle for $10 \sim 12 \mathrm{~h}$. To determine the effects of ATF6 on reporter activity, the ATF6 expression vector or empty vector (mock) was co-transfected with the reporter construct into the cells and cultured for $36 \mathrm{~h}$. After incubation under each condition, the cells were lysed and the luciferase activity in each lysate was measured using a Dual-Luciferase assay system (Promega). Reporter activity in each lysate was normalized to the co-transfected Renilla luciferase activity, and the results are shown as relative luciferase activity.

\section{Acknowledgements}

A part of this work was supported by the Ministry of Education, Culture, Sports, Science and Technology of Japan (Grant-in-Aid for Young Scientists (B), No. 21700403 to K.O.). 


\section{Author details}

'Department of Biomolecular Science, Faculty of Engineering, Gifu University, 1-1 Yanagido, Gifu 501-1193, Japan. ${ }^{2}$ Kazusa DNA Research Institute, 2-6-7 Kazusa-Kamatari, Kisarazu, Chiba 292-0818, Japan.

\section{Authors' contributions}

$\mathrm{KO}$ conceived of this study, carried out the molecular genetics studies, participated in the sequence alignment and drafted the manuscripts. HK, SI and KS participated in microarray analysis. YH and KK participated in its design and coordination. All authors read and approved the final manuscript.

Received: 29 July 2010 Accepted: 25 November 2010

Published: 25 November 2010

\section{References}

1. Trinklein ND, Aldred SF, Hartman SJ, Schroeder DI, Otillar RP, Myers RM: An abundance of bidirectional promoters in the human genome. Genome Res 2004, 14(1):62-66.

2. Li YY, YU H, Guo ZM, Guo TQ, Tu K, Li YX: Systematic analysis of head-tohead gene organization: evolutionary conservation and potential biological relevance. PLoS Comput Biol 2006, 2(7):e74.

3. Adachi N, Lieber MR: Bidirectional gene organization: a common architectural feature of the human genome. Cell 2002, 109(7):807-809.

4. Agirre X, Román-Gómez J, Vázquez I, Jiménez-Velasco A, Garate L, MontielDuarte C, Artieda P, Cordeu L, Lahortiga I, Calasanz MJ, Heiniger A, Torres A, Minna JD, Prósper F: Abnormal methylation of the common PARK2 and PACRG promoter is associated with downregulation of gene expression in acute lymphoblastic leukemia and chronic myeloid leukemia. Int $J$ Cancer 2006, 118(8):1945-1953.

5. Huang CC, Chang WS: Cooperation between NRF-2 and YY-1 transcription factors is essential for triggering the expression of the PREPL-C2ORF34 bidirectional gene pair. BMC Mol Biol 2009, 10:67.

6. Gaston $\mathrm{K}$, Fried $\mathrm{M}: \mathrm{YY} 1$ is involved in the regulation of the bi-directional promoter of the Surf-1 and Surf-2 genes. FEBS Lett 1994, 347(2-3):289-294

7. Zanotto $\mathrm{E}$, Shah $\mathrm{ZH}$, Jacobs $\mathrm{HT}$ : The bidirectional promoter of two genes for the mitochondrial translational apparatus in mouse is regulated by an array of CCAAT boxes interacting with the transcription factor NF-Y. Nucleic Acids Res 2007, 35(2):664-677.

8. Oh-hashi K, Koga H, Ikeda S, Shimada K, Hirata Y, Kiuchi K: CRELD2 is a novel endoplasmic reticulum stress-inducible gene. Biochem Biophys Res Commun 2009, 387(3):504-510.

9. Kim I, Xu W, Reed JC: Cell death and endoplasmic reticulum stress: disease relevance and therapeutic opportunities. Nat Rev Drug Discov 2008, 7(12):1013-1030.

10. Lindholm D, Wootz H, Korhonen L: ER stress and neurodegenerative diseases. Cell Death Differ 2006, 13(3):385-392.

11. Harding HP, Zhang $Y$, Ron D: Protein translation and folding are coupled by an endoplasmic-reticulum-resident kinase. Nature 1999, 397(6716):271-274.

12. Calfon M, Zeng H, Urano F, Till JH, Hubbard SR, Harding HP, Clark SG, Ron D: IRE1 couples endoplasmic reticulum load to secretory capacity by processing the XBP-1 mRNA. Nature 2002, 415(6867):92-96.

13. Zhu C, Johansen FE, Prywes R: Interaction of ATF6 and serum response factor. Mol Cell Biol 1997, 17(9):4957-4966.

14. Burda P, Jakob CA, Beinhauer J, Hegemann JH, Aebi M: Ordered assembly of the asymmetrically branched lipid-linked oligosaccharide in the endoplasmic reticulum is ensured by the substrate specificity of the individual glycosyltransferases. Glycobiology 1999, 9(6):617-625.

15. Ron D, Habener JF: CHOP, a novel developmentally regulated nuclear protein that dimerizes with transcription factors C/EBP and LAP and functions as a dominant-negative inhibitor of gene transcription. Genes Dev 1992, 6(3):439-453.

16. Ohoka N, Yoshii S, Hattori T, Onozaki K, Hayashi H: TRB3, a novel ER stressinducible gene, is induced via ATF4-CHOP pathway and is involved in cell death. EMBO J 2005, 24(6):1243-1255.

17. Kokame K, Agarwala KL, Kato H, Miyata T: Herp, a new ubiquitin-like membrane protein induced by endoplasmic reticulum stress. I Biol Chem 2000, 275:32846-32853.

18. Yoshida H, Haze K, Yanagi H, Yura T, Mori K: Identification of the cis-acting endoplasmic reticulum stress response element responsible for transcriptional induction of mammalian glucose-regulated proteins. Involvement of basic leucine zipper transcription factors. J Biol Chem 1998, 273(50):33741-33749.

19. Li M, Baumeister P, Roy B, Phan T, Foti D, Luo S, Lee AS: ATF6 as a transcription activator of the endoplasmic reticulum stress element: thapsigargin stress-induced changes and synergistic interactions with NF-Y and YY1. Mol Cell Biol 2000, 20(14):5096-5106.

20. Donati G, Imbriano C, Mantovani R: Dynamic recruitment of transcription factors and epigenetic changes on the ER stress response gene promoters. Nucleic Acids Res 2006, 34(10):3116-3127.

21. Oettgen P: Regulation of vascular inflammation and remodeling by ETS factors. Circ Res 2006, 99(11):1159-1166.

22. Chung SW, Chen YH, Perrella MA: Role of Ets-2 in the regulation of heme oxygenase-1 by endotoxin. J Biol Chem 2005, 280(6):4578-4584.

23. Shi Y, Seto E, Chang LS, Shenk T: Transcriptional repression by YY1, a human GLI-Kruppel-related protein, and relief of repression by adenovirus E1A protein. Cell 1991, 67(2):377-388.

24. Bossone SA, Asselin C, Patel AJ, Marcu KB: MAZ, a zinc finger protein, binds to C-MYC and $C 2$ gene sequences regulating transcriptional initiation and termination. Proc Natl Acad Sci USA 1992, 89(16):7452-7456.

25. Jones $\mathrm{PL}$, Ping $\mathrm{D}$, Boss JM: Tumor necrosis factor $\alpha$ and interleukin- $1 \beta$ regulate the murine manganese superoxide dismutase gene through a complex intronic enhancer involving C/EBP- $\beta$ and NF-KB. Mol Cell Biol 1997, 17(12):6970-6981

26. Uwanogho DA, Yasin SA, Starling B, Price J: The intergenic region between the Mouse Recql4 and Lrrc14 genes functions as an evolutionary conserved bidirectional promoter. Gene 2010, 449(1-2):103-117.

27. Chen PY, Chang WS, Lai YK, Wu CW: c-Myc regulates the coordinated transcription of brain disease-related PDCD10-SERPINI1 bidirectional gene pair. Mol Cell Neurosci 2009, 42(1):23-32.

28. Christophe-Hobertus C, Christophe D: Delimitation and functional characterization of the bidirectional THOX-DUOXA promoter regions in thyrocytes. Mol Cell Endocrinol 2010, 317(1-2):161-167.

29. Voth H, Oberthuer A, Simon T, Kahlert Y, Berthold F, Fischer M: Coregulated expression of HAND2 and DEIN by a bidirectional promoter with asymmetrical activity in neuroblastoma. BMC Mol Biol 2009, 10:28.

30. Zanotto E, Lehtonen V, Jacobs HT: Modulation of Mrps12/Sarsm promoter activity in response to mitochondrial stress. Biochim Biophys Acta 2008, 1783(12):2352-2362.

31. Grubenmann $C E$, Frank $C G$, Kjaergaard $S$, Berger EG, Aebi M, Hennet $T$ : ALG12 mannosyltransferase defect in congenital disorder of glycosylation type lg. Hum Mol Genet 2002, 11(19):2331-2339.

32. Chantret I, Dupré T, Delenda C, Bucher S, Dancourt J, Barnier A, Charollais A Heron D, Bader-Meunier B, Danos O, Seta N, Durand G, Oriol R, Codogno P, Moore SE: Congenital disorders of glycosylation type Ig is defined by a deficiency in dolichyl-P-mannose:Man7GlcNAc2-PP-dolichyl mannosyltransferase. J Biol Chem 2002, 277(28):25815-25822

33. Robinson SW, Morris CD, Goldmuntz E, Reller MD, Jones MA, Steiner RD, Maslen CL: Missense mutations in CRELD1 are associated with cardiac atrioventricular septal defects. Am J Hum Genet 2003, 72(4):1047-1052.

34. Rupp PA, Fouad GT, Egelston CA, Reifsteck CA, Olson SB, Knosp WM, Glanville RW, Thornburg KL, Robinson SW, Maslen CL: Identification, genomic organization and mRNA expression of CRELD1, the founding member of a unique family of matricellular proteins. Gene 2000, 293(12):47-57.

35. Maslen CL, Babcock D, Redig JK, Kapeli K, Akkari YM, Olson SB: CRELD2: gene mapping, alternate splicing, and comparative genomic identification of the promoter region. Gene 2006, 382:111-120.

36. Ortiz JA, Castillo M, del Toro ED, Mulet J, Gerber S, Valor LM, Sala S, Sala F, Gutiérrez LM, Criado M: The cysteine-rich with EGF-like domains 2 (CRELD2) protein interacts with the large cytoplasmic domain of human neuronal nicotinic acetylcholine receptor $\mathrm{a} 4$ and $\beta 2$ subunits. J Neurochem 2005, 95(6):1585-1596.

37. Nundlall S, Rajpar MH, Bell PA, Clowes C, Zeeff LA, Gardner B, Thornton DJ, Boot-Handford RP, Briggs MD: An unfolded protein response is the initial cellular response to the expression of mutant matrilin- 3 in a mouse model of multiple epiphyseal dysplasia. Cell Stress Chaperones 2010, 15(6):835-849.

doi:10.1186/1471-2164-11-664

Cite this article as: Oh-hashi et al:: Role of an ER stress response element in regulating the bidirectional promoter of the mouse CRELD2 - ALG12 gene pair. BMC Genomics 2010 11:664. 